\title{
Laparoscopic versus Open Complete Mesocolic Excision and D3 Lymphadenectomy in Right Colon Carcinoma
}

\author{
Ali Zedan Tohamy ${ }^{1 *}$, Hanan A. Eltyb², Marwa T. Hussien ${ }^{3}$, Haisam Atta ${ }^{4}$
}

*Corresponding author: Dr. Ali Zedan Tohamy South Egypt Cancer Institute Assiut University, Assiut, Egypt Telephone: +201006367475 E-mail: Alizedan73@yahoo.com

\section{Abbreviations:}

CME: complete mesocolic excision; LCME: laparoscopic complete mesocolic excision; OCME: open complete mesocolic excision;

TME: total mesorectal excision; CVL: central vascular ligation;

OS: overall survival;

DFS: disease-free survival.
Received: 16.10 .2020

Accepted: 10.12 .2020

Copyright ( C Celsius Publishing House www.sgo-iasgo.com
'Surgical Oncology Department, South Egypt Cancer Institute, Assiut University, Assiut, Egypt ${ }^{2}$ Medical Oncology Department, South Egypt Cancer Institute, Assiut University, Assiut, Egypt ${ }^{3}$ Department of oncologic pathology, South Egypt Cancer Institute, Assiut University, Assiut, Egypt ${ }^{4}$ Department Diagnostic Radiology, South Egypt Cancer Institute, Assiut University, Assiut, Egypt

\section{ABSTRACT}

Background and objectives: Complete mesocolic excision (CME) technique seems to be gaining popularity in cancer colon management. We aim to compare the laparoscopic approach for CME with the open approach in right colon cancer treatment regarding the feasibility, safety and perioperative and oncologic outcomes.

Patients and methods: A prospective study which included all patients that underwent radical right hemicolectomy for pathologic confirmed stage II or stage III tumor with CME at South Egypt Cancer Institute, Assiut University from January 2012 to December 2019. Patients were grouped according to the surgical approach into the laparoscopic colectomy (LCME) group $(n=48)$ or the open colectomy (OCME) group $(n=48)$.

Results: The mean operative time was significantly longer in the LCME group than in the OCME group with less mean intraoperative blood loss. The conversion was required in 4 patients $(8.3 \%)$ in the LCME group. The use of laparoscopy increased the number of harvested lymph nodes than in the open approach $(39.81 \pm 16.74$ vs $32.65 \pm 12.28$, respectively, $\mathrm{P}=0.010$ ). The laparoscopic approach was associated with a shorter time interval to first flatus and shorter time interval to liquid and normal diet after surgery. The postoperative hospital stay was significantly shorter in the LCME group ( $9.13 \pm 1.57$ day) than in the OCME group $(13.04 \pm 3.07$ day). The complication rate was slightly lower in the LCME (14.7\%) than in the OCME group (27.2\%), $(P=0.252)$. The 3-years OS in the LCME group was similar to that in OCME ( $78.2 \%$ vs $63.2 \%$, respectively, P-value $=0.423)$. The three years DFS in the laparoscopic group was higher (74.5\%) than the open group (60.0\%) but didn't reach statistical significance ( $\mathrm{P}$-value $=0.266)$.

Conclusions: In conclusion, laparoscopic CME right hemicolectomy is a technically feasible and safe procedure if surgeon expertise is present. LCME has long-term oncologic outcomes (recurrence and survival) comparable to open surgery for the management of patients with stage II or III colon cancer.

Key words: complete mesocolic excision (CME), laparoscopic colectomy, right colon, D3 lymphadenectomy, survival

\section{INTRODUCTION}

In 2009, Dr. Hohenberger was the first to propose the concept of complete mesocolic excision (CME) for colon cancer surgery (1) according to the concept 
of total mesorectal excision (TME) for rectal cancer. The technique of CME relies on three key components: (I) sharp dissection in the embryologic plane between the parietal fascia and visceral (mesenteric) fascia to remove mesentery together with its lymphatic drainage as an intact envelope (2); the principle behind this technique is to avoid any inadvertent exfoliation of the tumour cells from the mesentery into the peritoneal cavity; (II) proximal ligation of feeding vessels at their origin to remove apical lymph nodes and; (III) resection of a sufficient length of the bowel to remove potentially involved lymph nodes in a longitudinal direction (3).

After the $\mathrm{CME}$ technique postulation, $\mathrm{CME}$ with central vascular ligation (CVL) has been applied by many European centers for colon cancer (2). In Japan, Korea and several other Asian countries, a D3 lymphadenectomy technique, which has a concept close to $\mathrm{CME}$, has also gained popularity $(4,5)$. CME and D3 lymphadenectomy are associated with higher reported survival rates than conventional colon resection surgery (3). These results made many surgeons consider CME and D3 lymphadenectomy techniques as the standard of care for clinical stage II and III colon cancer surgery.

After the introduction of laparoscopic approach for colorectal surgery, several randomized trials (6-8) and systematic reviews $(9,10)$ have shown that the laparoscopic approach for colon cancer surgery is associated with faster recovery and less morbidity as compared to the standard open approach without affecting oncologic outcomes (11-13). Recently, several studies have discussed the feasibility of laparoscopic CME, primarily for right colon cancer with promising results $(9,14-17)$.

The aim of the present study is to compare the laparoscopic approach for CME with the open approach in right colon cancer treatment with regards to the feasibility, safety and perioperative and oncologic outcomes.

\section{PATIENTS AND METHODS}

This is a prospective study which included all patients that underwent radical right hemicolectomy for pathologic confirmed stage II or stage III tumour with CME at South Egypt Cancer Institute, Assiut University from January 2012 to December 2019. The exclusion criteria included colon cancer with stage IV disease, synchronous or previous malignancies and those with extra-colonic invasion (T4b). Moreover, nonmalignant cases and emergency cases with bowel obstruction or intestinal perforation were excluded from the study. The study was approved by the institutional review board (IRB). All enrolled patients were divided into the laparoscopic colectomy (LCME) group $(n=48)$ or the open colectomy (OCME) group $(n=48)$, depending on the surgical approach.

\section{We assigned the patients for the open and laparoscopic approach as follows}

At the beginning of the study, we did not have a speculum and had little laparoscopic surgery experience. Also, patients who had a history of many abdomen and pelvis operations were considered for an open colectomy. These are all the reasons that determine our choices.

The preoperative evaluation consisted of medical history evaluation, physical examination, basic laboratory studies including serum CEA, full preoperative colonoscopy, abdominal ultrasound, CT abdomen and pelvis, chest X-ray/CT chest and selective PET/CT.

Surgical outcome parameters, included incision length, operative time, blood loss, conversion rate, the postoperative pain score, postoperative first passage of flatus, hospital stay duration, and postoperative morbidity and mortality within 30 days after surgery.

Oncologic outcome parameters, including tumour size, distal, proximal and circumferential resection margins, number of lymph nodes retrieved, TNM classification were collected.

Follow-up data, including the use of chemotherapy, local recurrence rate, distant metastasis rate, and short-term survival data (including overall survival and recurrence-free survival rates) were collected.

\section{SURGICAL TECHNIQUE}

\section{Open approach}

Lateral-to-medial approach, starting via an incision of Toldt line, is used for the complete mobilization of the colon. The visceral and parietal fasciae are separated by sharp dissection to ensure an intact mesocolon. The feeding arteries are transected close to their origin from the superior mesenteric artery at the left side of SMV (central vascular tie). For cecal, ascending colon and hepatic flexure tumour, right hemicolectomy is performed with the division of the colon at midtransverse and division of the ileum $10 \mathrm{~cm}$ of the terminal ileum. The ileocolic, right colic (if present) and right branch of middle colic vessels are divided. For tumours distal to hepatic flexure, extended right hemicolectomy is performed with resection of proximal 
$2 \beta$ of the transverse colon and division of middle colic vessels at their origin (fig. 1a). For tumours of hepatic flexure and proximal transverse colon, a part of the greater omentum is removed en-block with the specimen. An end to end or end to side ileocolic anastomosis is performed using a hand-sewn technique with 3-0 vicryl suture.

\section{Laparoscopic approach}

The medial-to-lateral approach is used in all cases. Firstly, the mesentery at the junction of the terminal ileum and cecum is grabbed and pulled to the right lower quadrant to identify the ileocolic pedicle. The peritoneum on the caudal aspect is incised parallel to the arc of the ileocolic vessels and dissection proceeds to enter into the retroperitoneal plane. Then sharp dissection proceeds from medial to lateral and in caudal-cephalic direction to separate the posterior layer of mesocolon from the parietal fascia, exposing and protecting right gonadal vessels, ureter the duodenum, and head of the pancreas. This is followed by the dissections of the gastrocolic ligament, the right side of the greater omentum, and lateral peritoneum of the colon. The division of vessels is similar to that discussed in the open approach at their origin from superior mesenteric vessels (central vascular tie) (fig. 1b). The pedicle vessels are ligated with hemoclips, and divided using bipolar vessel-sealing devices. Bowel resection is similar to the open approach.

The specimen was extracted from a small midline incision (about $5 \mathrm{~cm}$ ) proximal to the umbilicus. Using a wound protector for extraction is recommended to minimize the risk of wound contamination and tumour spillage. An extra-corporeal end to end or end to side ileocolic anastomosis is performed using hand-sewn or stapling techniques (fig. 1c).

\section{Pathological examination}

Gross examination of the CME-colectomy specimen is done first to check for the resection quality (fig. 1d). Generally, six items are assessed: (1) morphologic assessment of the plane of dissection, (2) length of colon resected, (3) length of high tie vascular ligation of the mesenteric artery to the colon, and (4) the mesocolic area, (5) the shortest distance from the vascular high ligation point to the tumour and intestine and (6) number of lymph nodes harvested. The American Society of Clinical Oncology (ASCO) recommends that $>12$ lymph nodes are needed for accurate staging. Afterwards, the specimen was formalin-fixed paraffin-embedded (FFPE), forming tissue blocks. The blocks were cut into $5 \mu \mathrm{m}$-thick and mounted on glass
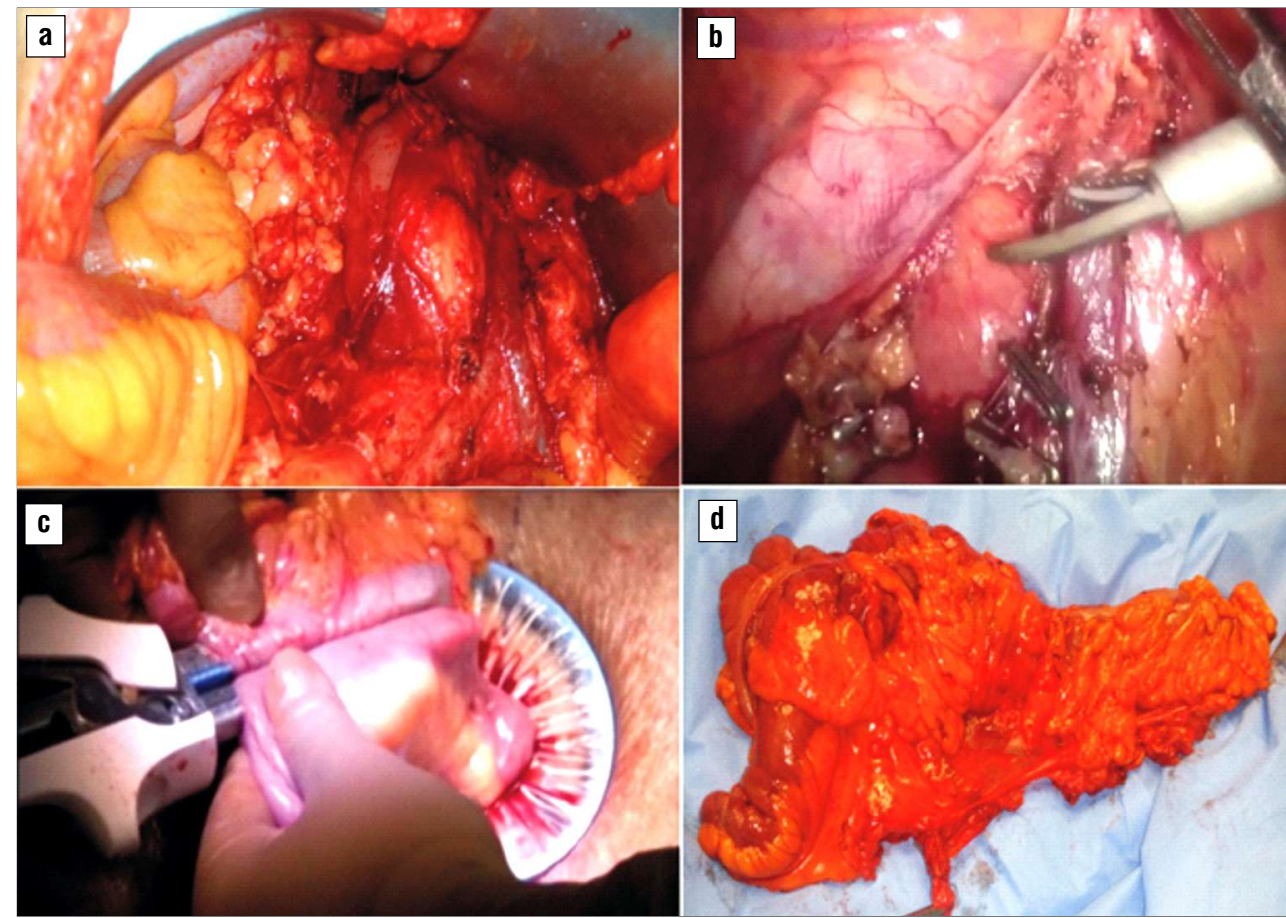

Figure1 - Surgical technique and pathologic examination. (a) Open CME with the division of feeding vessels at their origin, (b) Laparoscopic CME showing clipped ileocolic vessels at their origin from the superior mesenteric vessels, (c) Side-to-side stapled ileocolic anastomosis after LCME right hemicolectomy with the use of wound protector, (d) CME specimen for gross pathologic assessment before fixation 
slides. The slides were stained with hematoxylin and eosin stain for light microscopy evaluation. If we encountered difficulties in determining the tumour depth in T4 lesions, we performed double staining using Victoria blue and hematoxylin-eosin to identify the serosa of the colon.

\section{Follow-up and surveillance}

The patients will undergo a physical examination every three months in the first two years and once in six months for the following two years. After this period, there will be an annual examination for the next years. A chest $\mathrm{X}$-ray and abdominopelvic computed tomography (CT) scan and serum carcinoembryonic antigen (CEA) assay is performed every six months for the first two years and yearly thereafter. A colonoscopy is performed after one year from the surgery, and then after three years.

\section{Statistical methods}

SPSS version 23.0 was used for data management. Mean and standard deviation described quantitative data, the qualitative data was counted with percentages. Quantitative data were compared using t-test and qualitative data using Chi-square/Fisher exact tests. Overall survival was calculated from the date of pathologic diagnostic confirmation to death or last follow-up. The disease-free survival was calculated from the date of curative surgery up to first evidence of either local recurrence or distant metastasis or both. Kaplan Meier methods were used to estimate survival and log-rank test to compare curves with P-value always 2 tailed and significant at 0.05 level.

\section{RESULTS}

This study included the total number of 96 patients (54 males and 42 females), divided into the laparoscopic colectomy ( $L C M E)$ group $(n=48)$ or the open colectomy (OCME) group $(n=48)$ depending on the surgical approach. Regarding the patients' demographic characteristics, there were no statistically significant differences between the 2 groups regarding age, gender or comorbidities. Mean age was $61.56 \pm 13.52$ in OCME and $59.53 \pm 10.92$ in LCME ( $p=0.642)$. Concerning the pathologic parameters, the differences as regard to tumour location, tumour differentiation, TNM stage were statistically insignificant between the two groups. The most common tumour location in both groups was the ascending colon (67 patients). Moderatelydifferentiated tumors were more prevalent in both groups. Stage III was the final pathologic stage in 55 patients vs 41 cases with stage II (table 1).

\section{Operative data and technical aspect according to surgical approach}

The mean length of the specimen in the open group was about $27.3 \pm 3.2 \mathrm{~cm}$, which is significantly longer than the laparoscopic group $(20.1 \pm 1.97 \mathrm{~cm} ; \mathrm{P}<0.001)$. Mean tumour size was $5.6 \pm 0.9$ in OCME vs $4.5 \pm 0.7$ in LCME with $P<0.001$. Similarly, the mesentery area was

Table 1 - Tumour site, pathology, TNM stage \& co-morbidities

\begin{tabular}{|c|c|c|c|c|c|c|}
\hline & & \multicolumn{4}{|c|}{ Surgical approach } & \multirow[t]{3}{*}{$\mathrm{P}$-value * } \\
\hline & \multirow[b]{2}{*}{ Count } & \multicolumn{2}{|c|}{ Open surgery } & \multicolumn{2}{|c|}{ Laparoscopic } & \\
\hline & & $\%$ & Count & $\%$ & & \\
\hline Tumour locations & $\begin{array}{l}\text { Ascending colon } \\
\text { Cecum } \\
\text { Hepatic flexure }\end{array}$ & $\begin{array}{c}33 \\
8 \\
7\end{array}$ & $\begin{array}{l}68.8 \% \\
16.7 \% \\
14.6 \% \\
\end{array}$ & $\begin{array}{c}34 \\
12 \\
2\end{array}$ & $\begin{array}{c}70.8 \% \\
25.0 \% \\
4.2 \%\end{array}$ & 0.188 \\
\hline Pathological type & $\begin{array}{l}\text { Well-differentiated } \\
\text { Moderately-differentiated } \\
\text { Poorly-differentiated }\end{array}$ & $\begin{array}{c}6 \\
29 \\
13\end{array}$ & $\begin{array}{l}12.5 \% \\
60.4 \% \\
27.1 \%\end{array}$ & $\begin{array}{c}9 \\
31 \\
8\end{array}$ & $\begin{array}{l}18.8 \% \\
64.6 \% \\
16.7 \%\end{array}$ & 0.451 \\
\hline TNM stage & $\begin{array}{l}\text { II } \\
\text { III }\end{array}$ & $\begin{array}{l}19 \\
29\end{array}$ & $\begin{array}{l}39.6 \% \\
60.4 \%\end{array}$ & $\begin{array}{l}22 \\
26\end{array}$ & $\begin{array}{l}45.8 \% \\
54.2 \%\end{array}$ & 0.680 \\
\hline Potential comorbidities & $\begin{array}{l}\text { Hypertension } \\
\text { Cerebrovascular disease } \\
\text { Coronary disease } \\
\text { Diabetes } \\
\text { Pulmonary insufficiency }\end{array}$ & $\begin{array}{c}22 \\
2 \\
7 \\
10 \\
7\end{array}$ & $\begin{array}{c}45.8 \% \\
4.2 \% \\
14.6 \% \\
20.8 \% \\
14.6 \% \\
\end{array}$ & $\begin{array}{c}23 \\
2 \\
5 \\
11 \\
7\end{array}$ & $\begin{array}{c}47.9 \% \\
4.2 \% \\
10.4 \% \\
22.9 \% \\
14.6 \%\end{array}$ & 0.984 \\
\hline
\end{tabular}

${ }^{*} \mathrm{P}$-value is significant $\leq 0.05$ 
higher in OCME than LCME $\left(15.36 \mathrm{~mm}^{2}\right.$; vs $14.22 \mathrm{~mm}^{2}$, respectively) with $P<0.001$. Sufficient proximal and distal resection margins were obtained in both groups. Patients from the laparoscopic group had a shorter incision, with mean length 6.10 vs $18.79 \mathrm{~cm}$.

The mean operative time was significantly longer in the LCME group than in the OCME group with less mean intraoperative blood loss. There was a greater distance between the tumour and high tie in the LCME group with greater distance between nearest bowel wall and high tie. The use of laparoscopy increased the number of harvested lymph nodes than the open approach (39.81 \pm 16.74 vs $32.65 \pm 12.28$, respectively, $\mathrm{P}=0.010$ ). There was no difference achieving an intact mesocolic plane in the laparoscopic group vs the open group (77.1\%\% vs $83.3 \% \mathrm{P}=0.609$ ), (table 2 ).

\section{Post-operative data according to surgical approach}

Postoperatively, patients in the LCME group had lower pain scores than the OCME group $(3.25 \pm 0.76$ vs $5.31 \pm 0.88, \mathrm{P}<0.001)$. The laparoscopic approach was associated with a shorter time interval to first flatus ( 56.77 hs vs 100.48 hs, $\mathrm{P}<0.001$ ). The LCME patients had a shorter time interval to a liquid diet and a shorter time interval to a normal diet after surgery. The postoperative hospital stay was significantly shorter in the LCME group $(9.13 \pm 1.57$ day) than in the OCME group (13.04 \pm 3.07 day), $\mathrm{P}<0.001$ (table 3 ).

\section{Post-operative morbidity}

The complication rate was lower in the LCME (14.7\%) than the OCME group (27.2\%), ( $P=0.252)$. There was also no significant difference between both groups in the incidence of wound infection, lymphatic leakage, anastomotic leakage, pulmonary infection, ileus, deep venous thrombosis and 30-days mortality. The conversion rate was required in four patients (8.3\%) in the LCME group (table 4). The reasons for conversion were technically difficult dissection in two patients,

Table 2 - Operative data and technical aspect according to surgical approach

\begin{tabular}{|c|c|c|c|c|c|}
\hline & \multicolumn{4}{|c|}{ Surgical approach } & \multirow[t]{3}{*}{ P-value * } \\
\hline & \multicolumn{2}{|c|}{ Open surgery } & \multicolumn{2}{|c|}{ Laparoscopic } & \\
\hline & Mean & SD & Mean & SD & \\
\hline Length of surgical specimen $(\mathrm{cm})$ & 27.33 & 3.22 & 20.10 & 1.97 & $<0.001$ \\
\hline Tumour size $(\mathrm{cm})$ & 5.63 & .914 & 4.50 & 0.715 & $<0.001$ \\
\hline Area of mesentery $\left(\mathrm{mm}^{2}\right)$ & 15.36 & 1.24 & 14.22 & 0.91 & $<0.001$ \\
\hline Proximal resection margin $(\mathrm{cm})$ & 20.27 & 8.821 & 16.58 & 5.870 & 0.018 \\
\hline Distal resection margin $(\mathrm{cm})$ & 13.44 & 1.809 & 14.83 & 1.894 & $<0.001$ \\
\hline Incision length $(\mathrm{cm})$ & 18.79 & 3.18 & 6.10 & 0.93 & $<0.001$ \\
\hline Operative time (min) & 152.04 & 27.24 & 201.31 & 57.80 & $<0.001$ \\
\hline Blood loss (mL) & 264.17 & 67.70 & 189.33 & 74.22 & $<0.001$ \\
\hline No. of LNs retrieved & 32.65 & 12.28 & 39.81 & 16.74 & 0.010 \\
\hline Distance Tumour to high tie (mm) & 124.44 & 18.61 & 140.42 & 16.78 & $<0.001$ \\
\hline Distance from the nearest bowel wall to high tie $(\mathrm{mm})$ & 79.96 & 8.84 & 110.71 & 12.36 & $<0.001$ \\
\hline
\end{tabular}

${ }^{*} P$-value is significant $\leq 0.05$

Table 3 - Post-operative data according to surgical approach

\begin{tabular}{lccccc}
\hline & \multicolumn{2}{c}{ Surgical approach } & \multicolumn{2}{c}{ P-value * } \\
\hline & \multicolumn{2}{c}{ Open surgery } & \multicolumn{2}{c}{ Laparoscopic } \\
\hline Pain scores & Mean & SD & Mean & SD \\
\hline Getting out-of-bed time(days) & 5.31 & 0.88 & 3.25 & 0.76 & $<0.001$ \\
\hline First passage of flatus (hours) & 3.42 & 0.94 & 1.67 & 0.66 & $<0.001$ \\
\hline Time to resume liquid diet (day) & 100.48 & 11.63 & 56.77 & 9.51 & $<0.001$ \\
\hline Time of normal diet (day) & 4.91 & 1.19 & 3.50 & 1.09 & $<0.001$ \\
\hline Duration of hospital stay (day) & 6.27 & 0.96 & 4.73 & 0.76 & $<0.001$ \\
\hline
\end{tabular}

${ }^{*}$-value is significant $\leq 0.05$ 
severe adhesion in one patient, and uncontrolled bleeding in one patient. One patient in the OCME group died due to pulmonary embolism while one patient in the LCME group died due to sepsis after anastomotic leakage. The two patients in the OCME group who developed anastomotic leakage were managed by peritoneal lavage and exteriorization of the ileocolic anastomosis.

\section{Recurrence rate}

Local recurrence occurred in two patients in each group. Distant metastasis occurred in seven patients, with no significant differences between the two groups (table 5). Out of these seven patients, four had liver metastases, two had lung metastases, and one patient developed peritoneal carcinomatosis.

\section{Survival data}

The 3-years overall survival (OS) in the whole group was $74.0 \% \pm 5.3$. The 3 -years OS in the LCME group was similar to that in the OCME $(78.2 \%$ vs $63.2 \%$, respectively, P-value $=0.423$ ). Disease-free survival (DFS) at 3 -years in all cases was $70.5 \% \pm 5.5$. The three years DFS in the laparoscopic group was higher $(74.5 \%)$ than in the open group $(60.0 \%)$ but didn't reach statistical significance $(\mathrm{P}$-value $=0.266)($ fig. 2$)$.

\section{DISCUSSION}

After postulation of CME by Hohenberger et al. (1), this technique has been widely practised in many highvolume centers for colorectal cancer surgery. Most colorectal surgeons believe that CME colectomies are superior to "traditional" ones in local recurrence

Table 4 - Post-operative complications

\begin{tabular}{|c|c|c|c|c|c|c|}
\hline & & \multicolumn{4}{|c|}{ Surgical approach } & \multirow[t]{3}{*}{ P-value * } \\
\hline & & \multicolumn{2}{|c|}{ Open surgery } & \multicolumn{2}{|c|}{ Laparoscopic } & \\
\hline & & Count & $\%$ & Count & $\%$ & \\
\hline Wound infection & $\begin{array}{l}\text { No } \\
\text { Yes }\end{array}$ & $\begin{array}{c}44 \\
4\end{array}$ & $\begin{array}{c}91.7 \\
8.3\end{array}$ & $\begin{array}{c}47 \\
1\end{array}$ & $\begin{array}{c}97.9 \\
2.1\end{array}$ & 0.362 \\
\hline Lymphatic leakage & $\begin{array}{l}\text { No } \\
\text { Yes }\end{array}$ & $\begin{array}{c}47 \\
1\end{array}$ & $\begin{array}{c}97.9 \\
2.1\end{array}$ & $\begin{array}{c}47 \\
1\end{array}$ & $\begin{array}{c}97.9 \\
2.1\end{array}$ & 1.00 \\
\hline Anastomotic leakage & $\begin{array}{l}\text { No } \\
\text { Yes }\end{array}$ & $\begin{array}{c}46 \\
2\end{array}$ & $\begin{array}{c}95.8 \\
4.2\end{array}$ & $\begin{array}{c}47 \\
1\end{array}$ & $\begin{array}{c}97.9 \\
2.1\end{array}$ & 0.557 \\
\hline Pulmonary infection & $\begin{array}{l}\text { No } \\
\text { Yes }\end{array}$ & $\begin{array}{c}44 \\
4\end{array}$ & $\begin{array}{c}91.7 \\
8.3\end{array}$ & $\begin{array}{c}47 \\
1\end{array}$ & $\begin{array}{c}97.9 \\
2.1\end{array}$ & 0.168 \\
\hline lleus & $\begin{array}{l}\text { No } \\
\text { Yes }\end{array}$ & $\begin{array}{c}40 \\
8\end{array}$ & $\begin{array}{l}83.3 \\
16.7\end{array}$ & $\begin{array}{c}46 \\
2\end{array}$ & $\begin{array}{c}97.9 \\
2.1\end{array}$ & $0.04^{*}$ \\
\hline Deep venous thrombosis & $\begin{array}{l}\text { No } \\
\text { Yes }\end{array}$ & $\begin{array}{c}45 \\
3\end{array}$ & $\begin{array}{c}93.8 \\
6.3\end{array}$ & $\begin{array}{c}47 \\
1\end{array}$ & $\begin{array}{c}97.9 \\
2.1\end{array}$ & 0.307 \\
\hline 30 days mortality & $\begin{array}{l}\text { No } \\
\text { Yes }\end{array}$ & $\begin{array}{c}47 \\
1\end{array}$ & $\begin{array}{c}97.9 \\
2.1\end{array}$ & $\begin{array}{c}47 \\
1\end{array}$ & $\begin{array}{c}97.9 \\
2.1\end{array}$ & 1.00 \\
\hline Conversion rate & $\begin{array}{l}\text { No } \\
\text { Yes }\end{array}$ & - & $\begin{array}{l}- \\
-\end{array}$ & $\begin{array}{c}44 \\
4\end{array}$ & $\begin{array}{c}91.7 \\
8.3\end{array}$ & NA \\
\hline
\end{tabular}

*significant NA: non-comparable

Table 5 - Recurrence (local and distant) depending on the surgical approach

\begin{tabular}{|c|c|c|c|c|c|c|}
\hline & & \multicolumn{4}{|c|}{ Surgical approach } & \multirow[t]{2}{*}{ P-value } \\
\hline & & \multicolumn{2}{|c|}{ Open surgery } & \multicolumn{2}{|c|}{ Laparoscopic } & \\
\hline & & Count & $\%$ & Count & $\%$ & \\
\hline Local recurrence & $\begin{array}{l}\text { No } \\
\text { Yes }\end{array}$ & $\begin{array}{c}46 \\
2\end{array}$ & $\begin{array}{c}95.8 \\
4.2\end{array}$ & $\begin{array}{c}46 \\
2\end{array}$ & $\begin{array}{c}95.8 \\
4.2\end{array}$ & 1.00 \\
\hline Distant metastasis & $\begin{array}{l}\text { No } \\
\text { Yes }\end{array}$ & $\begin{array}{c}44 \\
4\end{array}$ & $\begin{array}{c}91.7 \\
8.3\end{array}$ & $\begin{array}{c}45 \\
3\end{array}$ & $\begin{array}{c}93.8 \\
6.3\end{array}$ & 0.69 \\
\hline
\end{tabular}




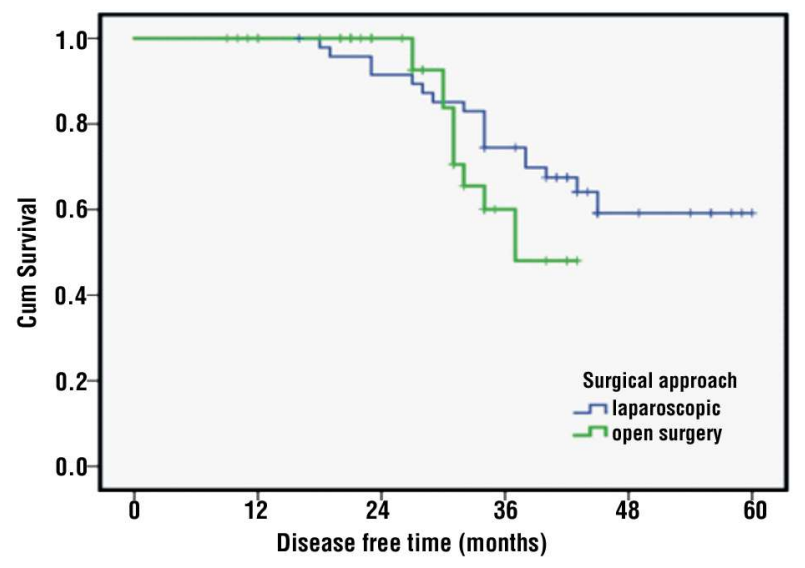

Figure 2 - Kaplan-Meier Survival Function Curve. It shows comparable survival rates between the $\mathbf{2}$ study groups regarding relapse-free survival rates

and cancer-related survival. The interest in last years focuses on decreasing the perioperative morbidity by using minimally-invasive approaches for the patient undergoing colon cancer surgery. Performing CME technique via laparoscopy aimed to reach the maximum oncologic quality of cancer surgery with least perioperative morbidity. Although the laparoscopic right hemicolectomy is widely performed globally, the feasibility and safety of laparos-copic CME have only recently been demonstrated in few centers $(14,15,18)$.

Some classic operative advantages of laparoscopic CME compared to open surgery include less blood loss and fewer transfusions $(7,8,19,20)$. Similarly, in our work, although the mean operative time was significantly longer in the LCME group than that in the OCME group, there was less mean intraoperative blood loss.

In this study, the recovery of gastrointestinal function after surgery in the laparoscopic approach was faster than OCME with a shorter time interval to first flatus, liquid diet and normal diet and less incidence of ileus. This is consistent with the solid concept about the prominent advantages of laparoscopic surgery in better GIT recovery (which has been reported in many large randomized trials as COST, CLASSIC, COLOR I, and $\operatorname{ALCCaS}$ trials) $(8,11,19,20)$. In addition, many authors compared LCME with OCME reported similar findings $(14,17)$. In a large meta-analysis, which investigated the outcome of LCME in 11 studies, they concluded that in four studies a shorter time interval to first flatus was reported with the laparoscopic approach, with a mean difference of 0.9 days. Also, the time to liquid diet (reported by 5 studies) was shorter for the LCME patients, with a mean difference of 1.84 days (16).
The overall complication rate was slightly lower in the LCME group (14.7\%) than the OCME group (27.2\%) in our work $(P=0.252)$. This was close to the data in a retrospective study reported by Wang et al., who showed that the short-term complication rate within 30 days was $16.3 \%$ (21). Also, Huang et al noted that in a retrospective analysis of 102 patients, the complications rates of LCME (4\%) and OCME (12\%) were comparable (22). Anastomotic leakage, pulmonary infection and deep venous thrombosis were higher in the open group than the laparoscopic group, despite not reaching statistical significance. There was no significant difference between both groups in the incidence of wound infection and lymphatic leakage. These data are supported by many authors' reports who showed comparable complication rates between open and laparoscopic approaches in CME technique $(14,17)$. Fortunately, major operative complications, e.g. duodenal injury, mesenteric vessel injury or ureteric injury, didn't occur in our study. We concluded that laparoscopic approach, if not decreasing morbidity, will have no further hazards to the patients.

The postoperative hospital stay in our study was significantly shorter in the LCME group (9.13 d) than in the OCME group (13.04d), $P<0.001$. This can be explained by early GIT recovery. An analysis of the short-term outcomes of colon and rectal laparoscopic resections revealed shorter hospitalization time (5-10) vs (6-11) $d, P=0.0011$ for laparoscopic procedures as in the COST, CLASSIC, COLOR I, and ALCCaS trials $(8,11$, $19,20)$. Huang et al. showed that length of hospital stay was significantly shorter in the LCME group than the OCME group ( $11 \pm 4 \mathrm{~d}$ vs $14 \pm 6 \mathrm{~d}$ ).

The mesentery area was higher in OCME than LCME (15.36 mm²; vs $14.22 \mathrm{~mm}^{2}$, respectively) with $\mathrm{P}<0.001$. In their meta-analysis of relevant studies, Negoi et al. reported the surface of the resected mesocolon to be larger in the LCME group ( $M D=11.75 \mathrm{~cm}^{2}, \mathrm{P}<0.001$ ), (16).

Our data showed a greater distance between the tumour and high tie in LCME group compared to OCME $(140.42 \pm 16.78$ vs $124.44 \pm 18.61, \mathrm{P}<0.001)$ as well as a greater distance between the nearest bowel wall and high tie $(110.71 \pm 12.36$ vs $79.96 \pm 8.84 \mathrm{P}<0.001)$. On the other hand, Gouvas and his colleague showed that distal ascending hepatic flexure, proximal transverse patients operated on through the open approach displayed significantly longer distances of tumour to high tie (open $11.67 \mathrm{~cm}$ vs laparoscopic $8.72 \mathrm{~cm}$; $P=0.049$ ) and of bowel wall to high tie (open $9.11 \mathrm{~cm}$ vs laparoscopic $6.5 \mathrm{~cm}, \mathrm{P}=0.015)$. However, all other groups (caecum, proximal ascending colon and left 
colon) showed comparable distance in terms of high tie to tumour and high tie to nearest bowel wall between laparoscopic and open groups (15).

In our work, we noted that laparoscopy increased the number of harvested lymph nodes than open approach $(39.81 \pm 16.74$ vs $32.65 \pm 12.28$, respectively, $\mathrm{P}=0.010)$. This is in a disagreement with many authors who showed that the numbers of harvested lymph nodes between the two groups were comparable (14, 17). In addition, in a large meta-analysis that included 11 studies, 10 of which reported the number of retrieved lymph nodes for 1376 LCME patients and 1271 OCME patients. They found no statistically significant mean difference between LCME and OCME (MD=-1.06, 95\%Cl: -3.65 to $1.53, \mathrm{P}=0.42$ ). These findings held true even after subgroup analysis according to the number of included patients (less or more than 100 patients in each group) and the geographical location of the study (Europe and Asia), (16).

In this study, the conversion was required in 4 patients in the LCME group (8.3\%). We regard this to be highly acceptable, as conversion rates in randomized controlled trials comparing laparoscopic and open colectomies ranged from $11 \%$ to $25 \%$. The leading cause of conversion in laparoscopic colectomy for cancer was local tumour progression $(7,8,23)$.

Local recurrence occurred in two patients in each group while distant metastasis occurred in seven patients, with no significant differences between the 2 groups. Port site metastasis didn't occur in our work. Sung et al. showed similar data with comparable overall recurrence rates among groups (LCME $12.9 \%$ vs OCME $20.0 \%, p=0.215$ ), (14).

Qin-Song et al. showed that the 2 groups of patients were also similar in terms of local recurrence rate (LCME $1.3 \%$ vs OCME 1.4\%), (17). In a large meta-analysis, 5 studies addressed the local recurrence rates in 1233 patients where there were no statistically significant differences between LCME and OCME (OR=0.67, 95\%Cl: 0.38 to $1.17, \mathrm{P}=0.16)$. On the other hand, the distant recurrence rate was addressed by four studies, with no statistically significant differences between the two groups (OR=0.98, 95\%Cl: 0.61 to $1.58, \mathrm{P}=0.94)$ ) (16).

In this study, found comparable 3-years OS and DFS among both groups. The 3 -years OS rate was $78.2 \% \pm 6.1$ in the laparoscopic group compared to $63.2 \% \pm 11.0$ in open surgery, P-value $=0.423$. The 3 -years DFS in the laparoscopic group was $74.5 \% \pm 6.4$ and in open surgery was $60.0 \pm 10.6$, P-value $=0.266$. In Negoi et al. metaanalysis, the 3 -years OS was reported by four studies, including 1010 patients. The laparoscopic approach was associated with a statistically significant better 3-years
OS, with an OR of 2.02 (95\%Cl: 1.31 to $3.12, \mathrm{P}=0.001)$, (16). In Qin-Song et al study, during the follow-up period (median 20.1 \pm 4.6 months), the laparoscopic and open groups were similar in terms of local recurrence rate $(1.3 \%$ vs $1.4 \%)$, distant metastasis rate $(1.3 \%$ vs $1.4 \%)$, and short-term survival rate $(79.5 \%$ vs $77.8 \%)$. These data are close to the results of our study (17). Sung et al. reported a median follow-up period of 58 months in the LCME group and 61 months in the OCME group. The 5-year OS rates of the LCME and OCME groups were 77.8 and $90.3 \%(p=0.028)$, respectively, whereas the 5-year DFS rates were 71.8 and $83.3 \%$ $(p=0.578)$, respectively $(14)$.

\section{CONCLUSION}

In conclusion, laparoscopic CME right hemicolectomy is a technically feasible and safe procedure if surgeon expertise is present. Despite the longer operation time, LCME has better short-term clinical outcomes (less operative blood loss, less postoperative pain, faster recovery of gastrointestinal function, shorter hospitalization time and higher lymph node yield). Moreover, LCME has long-term oncologic outcomes (recurrence and survival) comparable to open surgery for the management of patients with stage II or III colon cancer.

\section{Financial support and sponsorship}

We have not received any funding from any corporate body or pharmaceutical company.

\section{Conflicts of interest}

The authors have no conflicts of interest with regard to the contents of this work.

\section{Ethical Approval}

All procedures were carried out following the institutional committee's ethical standards and the 1964 Declaration of Helsinki. The study received the approval of the ethical committee of South Egypt Cancer Institute, Assiut University. The aim and steps of the study were explained to the patients, who signed written informed consents.

\section{REFERENCES}

1. Hohenberger W, Weber K, Matzel K, Papadopoulos T, Merkel S. Standardized surgery for colonic cancer: complete mesocolic excision and central ligation-technical notes and outcome. 
Colorectal disease. 2009:11(4):354-64.

2. Søndenaa K, Quirke P, Hohenberger W, Sugihara K, Kobayashi $H$, Kessler $\mathrm{H}$, et al. The rationale behind complete mesocolic excision (CME) and a central vascular ligation for colon cancer in open and laparoscopic surgery. International journal of colorectal disease. 2014;29(4):419-28.

3. West NP, Hohenberger W, Weber K, Perrakis A, Finan PJ, Quirke P. Complete mesocolic excision with central vascular ligation produces an oncologically superior specimen compared with standard surgery for carcinoma of the colon. Journal of clinical oncology. 2010;28(2):272-8.

4. Kotake K, Mizuguchi T, Moritani K, Wada O, Ozawa H, Oki I, et al Impact of D3 lymph node dissection on survival for patients with T3 and T4 colon cancer. International journal of colorectal disease. 2014;29(7):847-52.

5. Watanabe $\mathrm{T}$, Itabashi M, Shimada $\mathrm{Y}$, Tanaka S, Ito $\mathrm{Y}$, Ajioka $\mathrm{Y}$, Hamaguchi T, Hyodo I, Igarashi M, Ishida H, Ishiguro M. Japanese Society for Cancer of the Colon and Rectum (JSCCR) guidelines 2010 for the treatment of colorectal cancer. International journal of clinical oncology. 2012;17(1):1-29.

6. Bonjer HJ, Hop WC, Nelson H, Sargent DJ, Lacy AM, Castells A, et al. Laparoscopically assisted versus open colectomy for colon cancer: a meta-analysis. Arch Surg. 2007;142(3):298-303.

7. Guillou PJ, Quirke P, Thorpe H, Walker J, Jayne DG, Smith AM, et al. Short-term endpoints of conventional versus laparoscopic-assisted surgery in patients with colorectal cancer (MRC CLASICC trial) multicentre, randomised controlled trial. The lancet. 2005; 365(9472):1718-26.

8. Tinmouth J, Tomlinson G, Dalibon N, Moutafis M, Fischler M, Nelson $\mathrm{H}$, et al. Laparoscopically assisted versus open colectomy for colon cancer [1](multiple letters). New England Journal of Medicine. 2004; 351(9):933-4.

9. Bertelsen CA, Bols B, Ingeholm P, Jansen JE, Neuenschwander AU, Vilandt J. Can the quality of colonic surgery be improved by standardization of surgical technique with complete mesocolic excision?. Colorectal disease. 2011;13(10):1123-9.

10. Ma $\mathrm{Y}$, Yang Z, Qin H, Wang Y. A meta-analysis of laparoscopy compared with open colorectal resection for colorectal cancer Medical oncology. 2011;28(4):925-33.

11. Buunen M. Colon Cancer Laparoscopic or Open Resection Studfy Group. Survival after laparoscopic surgery versus open surgery for colon cancer: long-term outcomes of a randomised clincal trial. Lancet Oncol.. 2009;10:44-52.

12. Fleshman J, Sargent DJ, Green E, Anvari M, Stryker SJ, Beart Jr RW, et al. Clinical Outcomes of Surgical Therapy Study Group. Laparoscopic colectomy for cancer is not inferior to open surgery based on 5-year data from the COST Study Group trial. Annals of surgery. 2007;246(4):655-64

13. Jayne DG, Thorpe HC, Copeland J, Quirke P, Brown JM, Guillou PJ. Five-year follow-up of the Medical Research Council CLASICC trial of laparoscopically assisted versus open surgery for colorectal cancer. British journal of surgery. 2010;97(11):1638-45.

14. Bae SU, Saklani AP, Lim DR, Kim DW, Hur H, Min BS, et al. Laparoscopic-assisted versus open complete mesocolic excision and central vascular ligation for right-sided colon cancer. Annals of surgical oncology. 2014;21(7):2288-94.

15. Gouvas N, Pechlivanides G, Zervakis N, Kafousi M, Xynos E. Complete mesocolic excision in colon cancer surgery: a comparison between open and laparoscopic approach. Colorectal disease. 2012; 14(11):1357-64

16. Negoi I, Hostiuc S, Negoi RI, Beuran M. Laparoscopic vs open complete mesocolic excision with central vascular ligation for colon cancer: A systematic review and meta-analysis. World Journal of Gastrointestinal Oncology. 2017;9(12):475.

17. Sheng QS, Pan Z, Chai J, Cheng XB, Liu FL, Wang JH, et al. Complete mesocolic excision in right hemicolectomy: comparison between hand-assisted laparoscopic and open approaches. Annals of surgical treatment and research. 2017;92(2):90-6.

18. Li JC, Leung KL, Ng SS, Liu SY, Lee JF, Hon SS. Laparoscopicassisted versus open resection of right-sided colonic cancer - a prospective randomized controlled trial. International journal of colorectal disease. $2012 ; 27(1): 95-102$

19. Jayne DG, Guillou PJ, Thorpe H, Quirke P, Copeland J, Smith AM, et al. Randomized trial of laparoscopic-assisted resection of colorectal carcinoma: 3-year results of the UK MRC CLASICC Trial Group. Journal of Clinical Oncology. 2007;25(21):3061-8.

20. McCombie AM, Frizelle F, Bagshaw PF, Frampton CM, Hewett PJ, McMurrick PJ, et al. The ALCCaS trial: a randomized controlled trial comparing quality of life following laparoscopic versus open colectomy for colon cancer. Diseases of the Colon \& Rectum. 2018; 61(10):1156-62.

21. Wang $Y$, Zhang C, Zhang D, Fu Z, Sun Y. Clinical outcome of laparos-copic complete mesocolic excision in the treatment of right colon cancer. World journal of surgical oncology. 2017;15(1):1-6.

22. Huang JL, Wei HB, Fang JF, Zheng ZH, Chen TF, Wei B, et al. Comparison of laparoscopic versus open complete mesocolic excision for right colon cancer. International journal of surgery. 2015;23:12-7.

23. Hewett PJ, Allardyce RA, Bagshaw PF, Frampton CM, Frizelle FA, Rieger NA, et al. Short-term outcomes of the Australasian randomized clinical study comparing laparoscopic and conventional open surgical treatments for colon cancer: the ALCCaS trial. Annals of surgery. 2008;248(5):728-38. 